\title{
BTK Protein Variant
}

National Cancer Institute

\section{Source}

National Cancer Institute. BTK Protein Variant. NCI Thesaurus. Code C134489.

A variation in the amino acid sequence of the tyrosine-protein kinase BTK protein. 